\title{
Methods of treating keratitis and thermal burns in the cornea at various locations and areas with ligand-based peptides
}

\author{
Tereshenko $A^{1}$, Temnov $A^{2}$, Trifanenkova $I^{1}, \operatorname{Kodunov} A^{1 *}$ and Sklifas $A^{3}$ \\ ${ }^{1} \mathrm{~S}$. Fyodorov Eye Microsurgery Federal State Institution, Russia \\ ${ }^{2}$ Moscow Institute of Physics and Technology, Russia \\ ${ }^{3}$ Institute of cell Biophysics, Russia
}

\begin{abstract}
Neovascularization of the cornea is one of the most serious complications that can arise as a result of disease or damage to the eye.

Aim: to study the reparative properties of peptide solutions on the cornea.

Materials and methods: The experiment was carried out on 20 rabbits in two stages:

First stage: treatment of central thermal burns of the cornea without injury to the limbal region

Second stage: treatment of peripheral thermal burns of the cornea with injury to the limbal region. The experimental groups underwent instillations with the peptide solution in the conjunctival cavity. The control groups were treated for thermal corneal burns by standard methods. The condition of all four groups of animals was monitored daily over a period of 14 days. After which, the follow up was carried out 3 times a week. On day 1, 3, 7, 14, and 30, the animals were removed from experimental conditions and the morphology of their respective corneas was evaluated.

Results: By the $30^{\text {th }}$ day, the corneal transparency in the experimental group was approaching the initial state and the corneal thickness had been restored. In the control group, the corneal transparency and thickness were lesser than the analogous results in the experimental group. In the experimental group, the structural integrity of the cornea was preserved while the endothelium showed a loss in cell density. In the control group there was formation of vascular cataracts and edema of the corneal stroma.
\end{abstract}

Conclusions: After evaluating the results statistically, it can be concluded that tissue restoration is a lot faster with the use of peptide solutions.

\section{Introduction}

The corneal epithelium is the biological and physical barrier that protects the internal structure of the eye from the external environment. The corneal transparency, and subsequently the visual acuity depends upon the integrity and functional activity of the epithelial layer. Acute eye injuries, such as damage to the epithelia, are acute inflammatory processes which can lead to the pathological proliferation of blood vessels and impairment in the optical properties of the eye.

Neovascularization in the cornea is one of the most serious complications that can arise as a result of an infectious agent or damage to the eye such as injuries, burns of various etiologies, keratitis, and dystrophic diseases of the cornea. Ulcers in the cornea are a common complication of an infectious keratitis. This inflammation and tissue damage is caused as a result of infections caused by bacteria, fungi, or parasites. This is the leading cause of blindness in the developing world. Treatment of infectious keratitis is a serious problem and is being researched upon extensively.

Neovascularization is often accompanied by clouding of the cornea by the formation of cataracts. Depending on the size and location of the cataract with respect to the visual axis, it can significantly reduce visual acuity or cause complete blindness [1].
As of 2001, the occurrence of vascularized cataracts in the eye in the Russian Federation constituted $25.4 \%$ of all cases related to eye injuries (Alekseev G. F., Bordyugova G. G.). In the developing world, this form of cataract is the leading cause of blindness.

\section{Therapeutic potential}

Existing methods of treatment do not provide sufficient prevention against neovascularization and corneal opacity. A number of studies using experimental models of induced corneal neovascularization aimed at improving the effectiveness of the treatment did not show promising results $[2,3]$. As a result, there is an active global effort in seeking an alternative treatment for the mentioned condition.

One of the promising techniques for the treatment of corneal neovascularization is the use of stem cell peptides derived from the bone marrow or the corneal limbus region. Studies conducted on animals have shown stem cell peptides derived from the bone marrow

*Correspondence to: Alexey Kodunov, "MNTK” eye microsurgery “im. Akad. S. N. Fedorov" of the Ministry of Health of Russia 248007, street S. Fedorova 5, Kaluga, Russia, E-mail: 7alex72007@mail.ru

Received: July 09, 2018; Accepted: July 20, 2018; Published: July 23, 2018 
or the corneal limbus region can significantly improve the results of burn treatments related to the cornea [4].

Obtaining peptides from stem cell cultures can potentially help in treating or preventing complications arising from thermal injuries and toxic keratitis in the eye. Purified and lyophilized solutions of such peptides containing paracrine factors could further be effective in the prevention of corneal neovascularization.

\section{Material and methods}

Thermal injury was chosen as the experimental model for this experiment. In contrast to chemical damages, thermal injury prevents the potential damage caused by the chemical neutralization of peptides by aggressive chemical agents to the cornea.

Research was carried out on 20 rabbits (40 eyes in total). The breed used was "Gray Chinchilla" that weighs between 2.5 to 3.2 kilograms. The animals were kept in a vivarium in separate cages and had free access to food and water. The animals were treated humanely throughout the experiment. The experiment was carried out in two stages. Each of the stages included 5 animals in the control group and 5 animals in the experimental group. Totalling 10 animals in each stage of the experiment.

$\mathbf{1}^{\text {st }}$ stage of research: included the treatment of the central thermal burn of the cornea without damaging the limbal region.

$2^{\text {nd }}$ stage of research: included the treatment of the peripheral thermal burn of the cornea with damage to the limbal region.

Comparison of the effectiveness between the treatment with peptide solutions and classical remedies was carried out on thermal burns in the central and paracentral regions with damage to the limbal zone. Depending on the location of the thermal burns, the experiment was divided into two groups. Group 1 with central burns (5 rabbits - 10 eyes) and Group 2 with paracentral burns (5 rabbits - 10 eyes). The rabbits in the experimental groups received treatment in the form of instillations of peptide solutions. Likewise, two control groups of 5 rabbits each were formed based on the location of thermal burns. They were treated with a solution of Vigamox $^{\circledR}$ (moxifloxacin) and Solcoseryl ${ }^{\circledR}$ gel. Prior to commencing the experiment, stem cells were isolated from the bone marrow of the rabbits.

The next (third) stage of the experiment consisted of treating keratitis. It was conducted on 8 rats (16 eyes) who were divided into two groups (control and experimental group).

The model for inducing toxic keratitis was created using the methods described by Tereshenko A.V, et al. [5]

After inducing keratitis, the experimental group was administered a 6-fold instillation of the peptide solution in the conjunctival cavity over 14 days. The control group underwent an antibacterial and keratoprotective therapy (eye drops) for 14 days. Moxifloxacin was administered 4 times a day for 7 days and Corneregel $^{\circledR} 4$ times a day for 14 days.

\section{Isolation of stem cells from the bone marrow}

Mesenchymal Stem Cells (MSCs) were procured from the pelvic bones of the rabbits. The mononuclear fraction of bone marrow cells was isolated using the standard solution Lymphocyte-H (Cedarlane, Canada) to set a density gradient. The mononuclear cell suspension was plated on petri dishes and cultivated in Dulbecco's Modified Eagle Medium (DMEM) with 10\% Fetal Bovine Serum (FBS).
To confirm that the isolated cells possessed properties displayed by MSCs, osteogenic, chondrogenic, and adipogenic differentiations were carried out using standard protocol [6]. Osteogenic differentiation (supplemented with $10 \% \mathrm{FBS}, 100 \mu \mathrm{M}$ dexamethasone, $0.1 \mathrm{mM}$ ascorbic acid, and $10 \mathrm{nM} \beta$-glycerophosphate) was confirmed by the presence of alkaline phosphatase in the cell culture using standard reagents (SigmaAldrich, USA). Chondrogenic differentiation (supplemented by $10 \%$ FBS, $100 \mu \mathrm{M}$ dexamethasone, and $0.1 \mu \mathrm{g} / \mathrm{mL}$ TGF- $\beta$ ) was confirmed with staining cells with the dye Alcian blue. Adipogenic differentiation (supplemented with 10\% FBS, $10 \mu \mathrm{M}$ dexamethasone, $10 \mu \mathrm{g} / \mathrm{mL}$ insulin, and $100 \mu \mathrm{g} / \mathrm{mL}$ IBMX) was confirmed by using the dye Oil Red-O.

\section{Obtaining peptides}

Once a monolayer was formed in the cell culture, fresh medium was added, and after 3 days was replaced by conditioned cultural medium in addition to an MSC lysate. The cell cultures were destroyed using physico-chemical methods adhering to the methods described in Patent 2391990 Published 20.06.2010 Bulletin no. 17, and Patent 2341270 Published 20.12.2008 Bulletin no. 35. Two cycles of frozen from "' from distilled water.

\section{Thermal burn}

The thermal burn was inflicted after the instillation of $0.5 \%$ solution of proxymetacaine in the conjunctival cavity as per our developed method (Figure 1).

A special device with a metal cylinder and flat base (diameter 4 $\mathrm{mm}$ ) connected to an alternating current source was used. The peak temperature obtained was $210^{\circ} \mathrm{C} \pm 5^{\circ} \mathrm{C}$ (Figure 1). Immediately after thermal exposure, $0.3 \%$ of Ofloxacin (Floxal) was applied to the lower lid.

At the first stage, thermal exposure was strictly carried out in the center of the cornea (Figure 2). At the second stage, thermal exposure was carried out as per the illustrations in the scheme shown in Figure 3.

Instillations of $0.1 \%$ Benzalkonium chloride solution on all the rats 4 times a day was carried out during the third stage of the experiment to

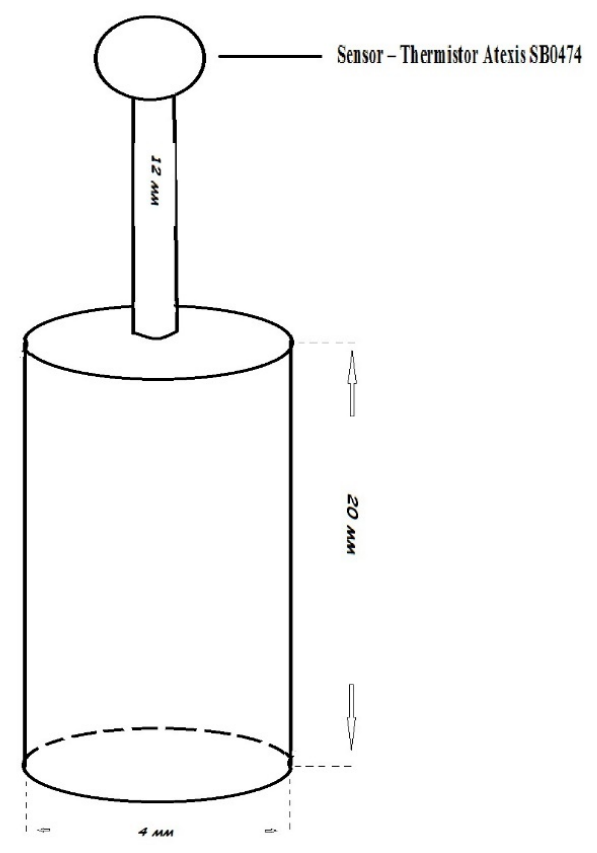

Figure 1. Device used to inflict thermal burns 


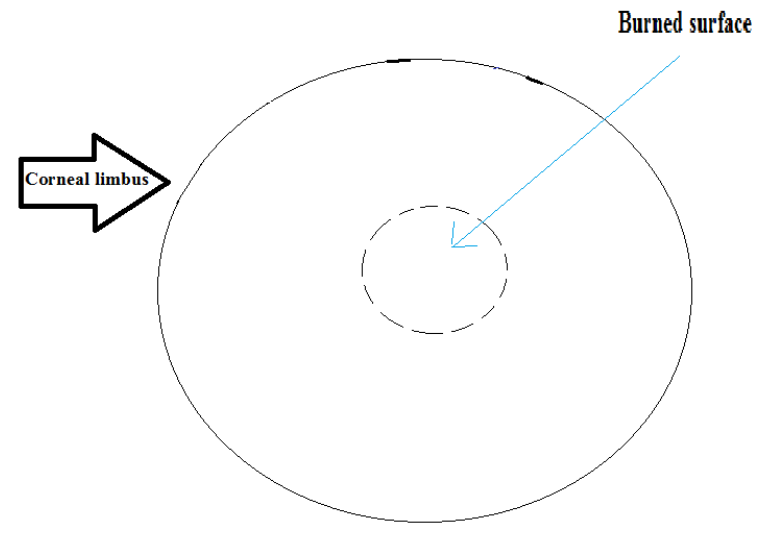

Figure 2. First stage thermal burn to the center of the cornea

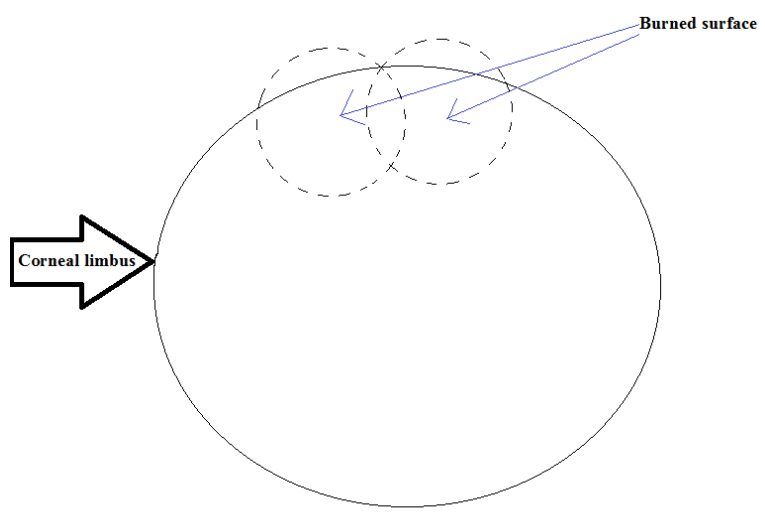

Figure 3. Second stage thermal burn to the periphery

induce keratitis with neovascularization in the cornea. On the $14^{\text {th }}$ day, the following was observed in all the eyes: chemosis, diffuse edema in all the layers of the cornea, a growth in the concentric limbal region 2 $\mathrm{mm}$ towards the optical zone.

On the first day after induction of thermal burns, all animals showed scarification in the form of scabs on the cornea after instillation with $0.5 \%$ proxymetacaine solution under a slit lamp HS (Haag-Streit International). Prior to scarification, photoregistration and evaluation of transparency was performed using corneal topography and corneal pachymetry. Images were taken using the camera Canon EOS 70D calibrated to the optical system of the slit lamp. On the third day, the lesion was stained and photographed for corneal examination using a Pentacam ${ }^{\circ}$ HR Typ 70900.

\section{Histological analysis}

Samples for histological analysis were collected on the $1^{\text {st }}, 3^{\text {rd }}, 7^{\text {th }}$, $14^{\text {th }}$, and $30^{\text {th }}$ day after induction of thermal burns. The corneal tissue was fixed in a $10 \%$ formalin neutral buffer and serial paraffin sections were prepared at a thickness of $3 \mu \mathrm{m}$.

\section{Treatment}

In the experimental group, the conjunctival cavity was instillated 6 times in an hour for the first 14 days and 4 times in an hour from the $14^{\text {th }}$ to the $30^{\text {th }}$ day. In the control group, treatment of thermal burns of the cornea was administered four times a day in the form of instillations of $0.5 \%$ solution of Vigamox (moxifloxacin) and applying Solcoseryl ${ }^{\circledR}$ gel on the lower eyelid during a 30-day time period.
The condition of animals belonging to all four groups was monitored daily for the first 14 days, followed by three times a week for the rest of the days. The following characteristics were evaluated clinically: the presence or absence of discharge, nature of the conjunctivital injection and its severity, lesion in the cornea (stained using $0.1 \%$ solution of fluorescent sodium), and the intensity of turbidity of the cornea $\left(\right.$ Pentacam $\left.^{\circledR}\right)$.

\section{Results}

The condition of the cornea immediately after inflicting thermal burns was not evaluated due to blepharospasms exhibited by animals in all four groups. In the experimental group, scarring in the cornea occurred on the first day (Figure 4)

On the third day, epithelialisation was observed in the corners of the burned area and granulation was observed at the centre of the affected areas. On the $7^{\text {th }}$ day (Figure 5), the lesion had completely epithelialized and no edema was observed in the surrounding tissues. A portion of the initial cornea was preserved in the centre of the lesion. In addition, the optical properties of the cornea remained impaired.

Significant improvement in the optical properties of the cornea was observed on the $14^{\text {th }}$ day. As per results obtained from corneal pachymetry, the thickness of the cornea was approaching normal values.

On the $30^{\text {th }}$ day (Figure 6), the transparency and the thickness of the cornea were almost fully restored, and the data from the pachymetry performed on the limbal region exceeded the initial values with an average of $20 \mu \mathrm{m}$.

In the control group on the first day (Figure 7), scarring was conducted in the cornea of the animals which had a more delicate consistency in comparison to the experimental group.

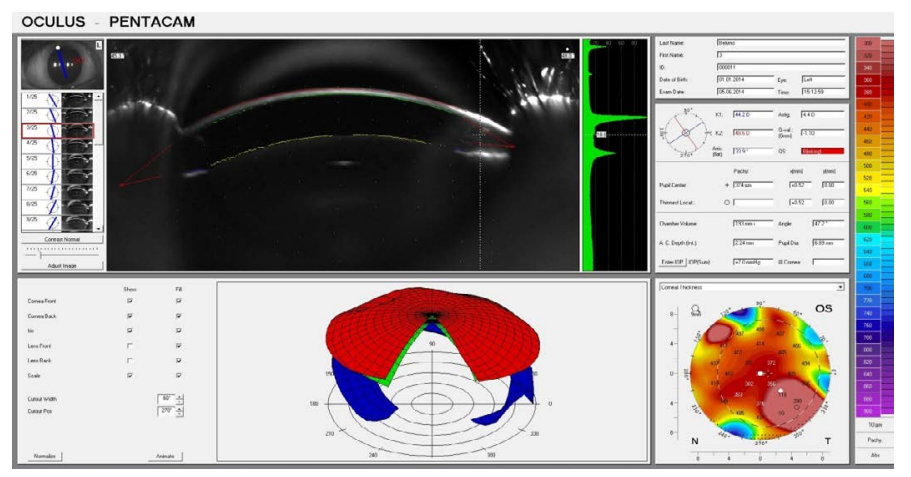

Figure 4. Experimental group: 1 day after thermal exposure

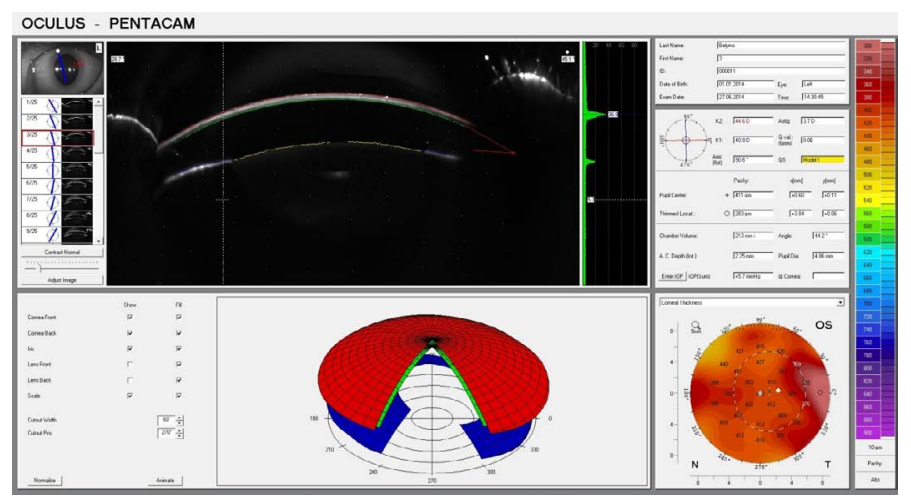

Figure 5. Experimental group: Seven days after thermal exposure 


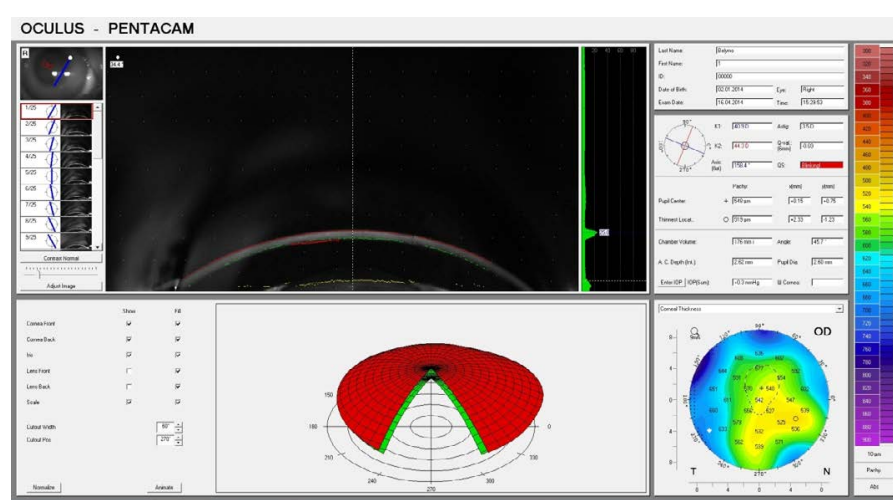

Figure 6. Experimental group: 30 days after thermal exposure

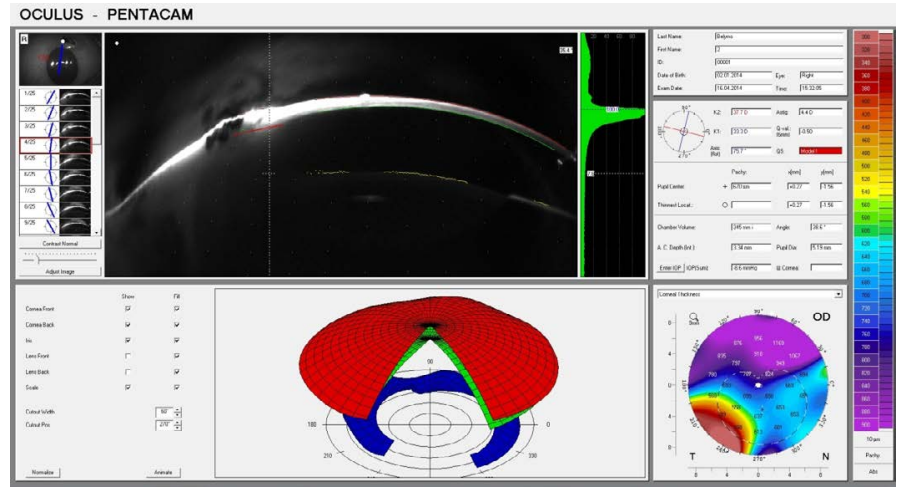

Figure 7. Control group: 1 day after thermal exposure

The lesion was stained on the third day. Epithelialisation had not begun in all corners (they were not seen in the outer region in one of the animals). Similarly, granulation in the centre of the lesion was not observed.

By the $7^{\text {th }}$ day (Figure 8 ), four of the rabbits exhibited complete epithelialisation and one rabbit showed the same by the $9^{\text {th }}$ day. The data from the Pentacam ${ }^{\circ}$ treatment revealed a significant reduction in the thickness of the cornea in the zone of the burn and severe impairment in the photoconductivity when compared to the experimental group.

On the $14^{\text {th }}$ day, the thickness and photoconductivity in the animals that no longer received instillations was worse than the experimental group.

On the $30^{\text {th }}$ day (Figure 9), the transparency and thickness of the cornea were lesser than the analogous results observed in the experimental groups as is depicted in Graph $1-3$ (Figure 10-12).

An increase in the transparency of the cornea (Figure 10) was measured by the Pentacam using arbitrary units. A value of 100 corresponds to complete opacity and subsequently 0 to complete transparency in the experimental region.

\section{Second stage of the experiment}

Evaluating the condition of the cornea during the first day after thermal burns was not possible due to significant blepharospasms exhibited in the eyes of animals in both the control and experimental groups (Figure 13,14).

On the third day, epithelialisation was observed in the corner of lesions belonging to animals in the experimental group. In the control group no such observation was noted. Data from the Pentacam ${ }^{\circledR}$ showed an increase in the thickness of the cornea between 10-30 $\mu \mathrm{M}$ (Graph 3) depending on the distance from the injured area.
In the experimental group, complete epithelialisation had occurred by the $5^{\text {th }}$ day (Figure 15). Furthermore, formation of new blood vessels in the cornea were observed (Figure 16).

By the $7^{\text {th }}$ day epithelialisation had terminated in both experimental and control groups. The corneal transparency was restored in practically all the experimental subjects. In the control group the transparency was not restored in the area that was affected by the thermal burn. The affected area in the limbus showed vascular growth and hyperpigmentation.

The overall condition of the eye in the experimental group by the $14^{\text {th }}$ day was close to the initial condition i.e. before administering the thermal burn. In the control group neoangiogenesis was observed in the cornea along with a persistent cataract.

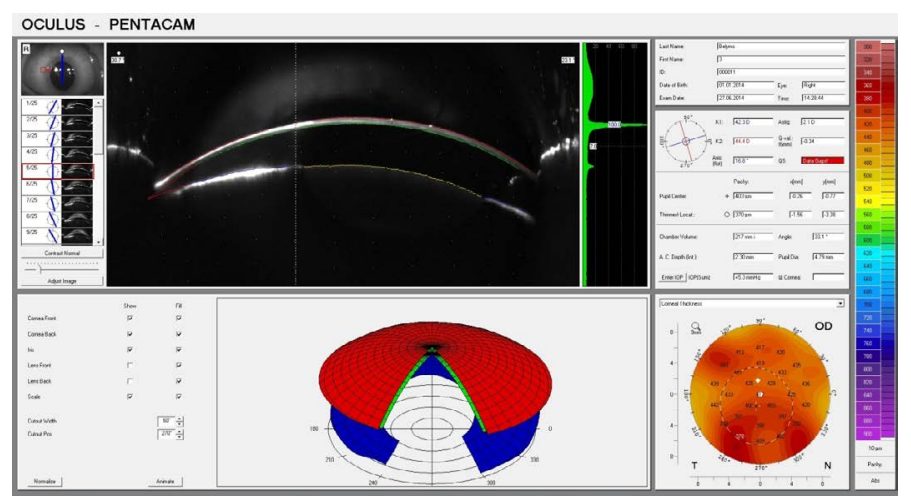

Figure 8. Control group: 7 days after thermal exposure

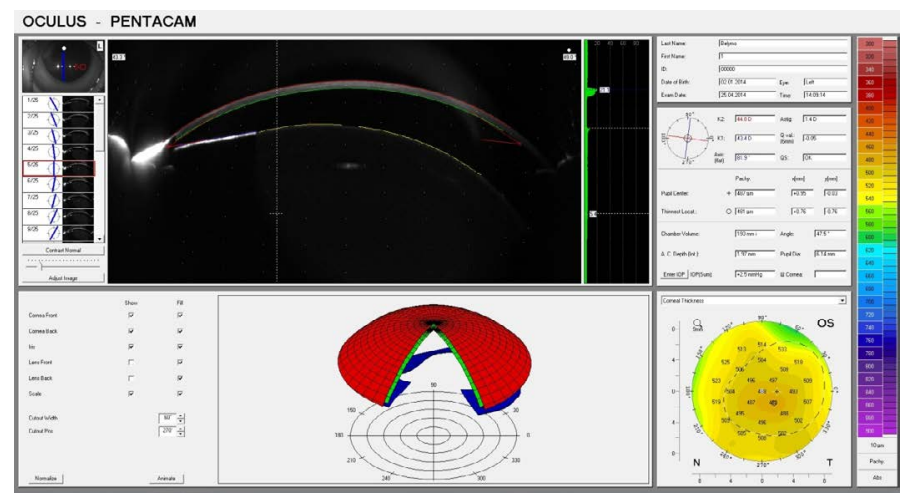

Figure 9. Control group: 30 days after thermal exposure

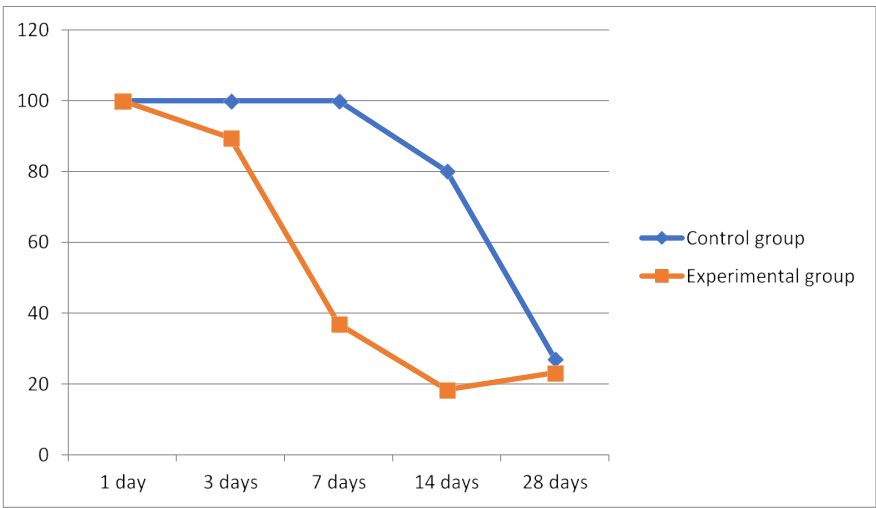

Figure 10. Graph 1 Dynamics of corneal transparency 


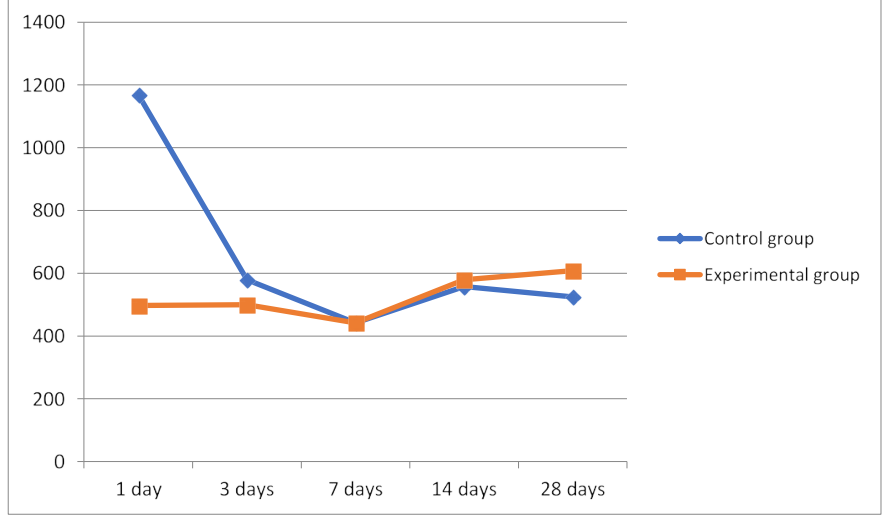

Figure 11. Graph 2 Dynamics of maximum corneal thickness

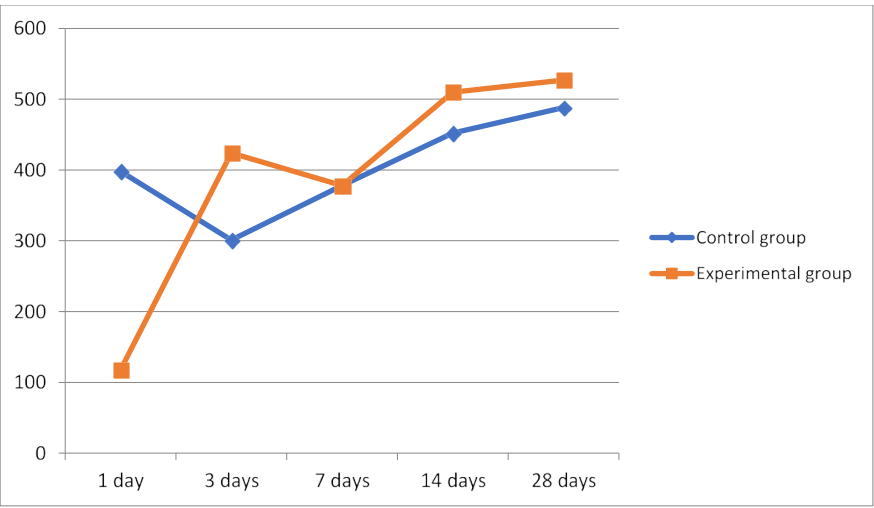

Figure 12. Graph 3 Dynamics of minimum corneal thickness

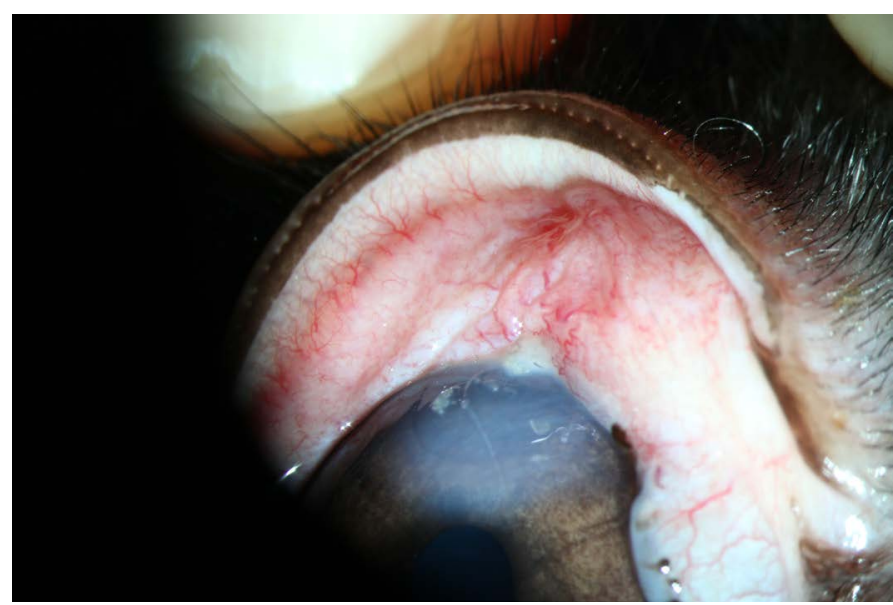

Figure 13. Experimental group: 1 day after thermal exposure

The effects of the thermal burn by the $30^{\text {th }}$ day in the experimental group presented themselves in the form of light stromal opacity in the centre of the burn (Figure 17). In the control group an increase in the vessel diameter in the cornea was observed (Figure 18). Similarly, the corneal transparency significantly improved, but did not match the initial state.

Results of the experiment were processed using the program Statistica 5.0. The parameters evaluated were: corneal transparency, maximum thickness of the cornea, and minimum thickness of the cornea.
It should be noted that the area adjacent to the thermal burn was taken as the maximum corneal thickness and the area at the center of the thermal burn as the minimum. As is shown in Graph 2 and 3 (Figure 11,12), the groups undergoing standard treatment showed edema in the areas surrounding injury. The most severe edema was observed during the first day. On the other hand, during the second phase, the animals in the experimental group showed an insignificant

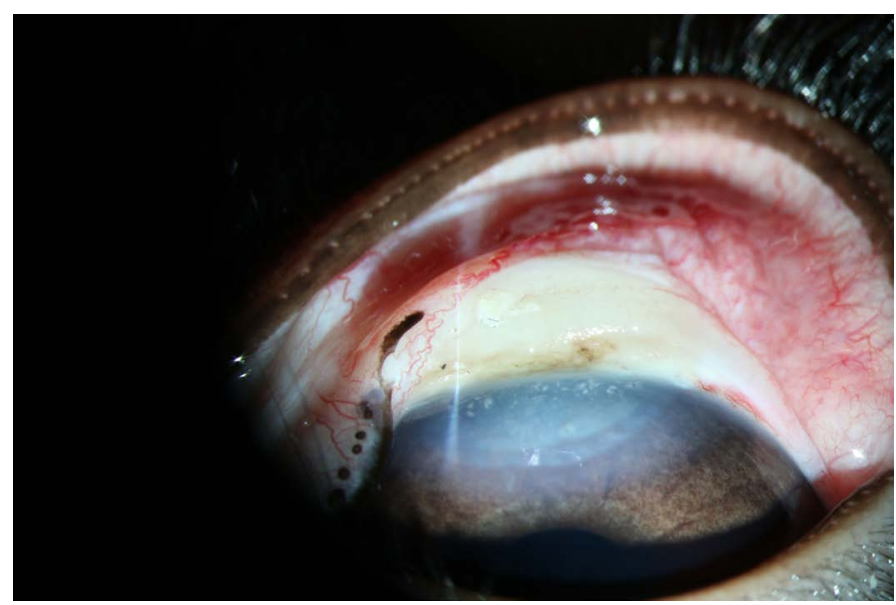

Figure 14. Control group: 1 day after thermal exposure

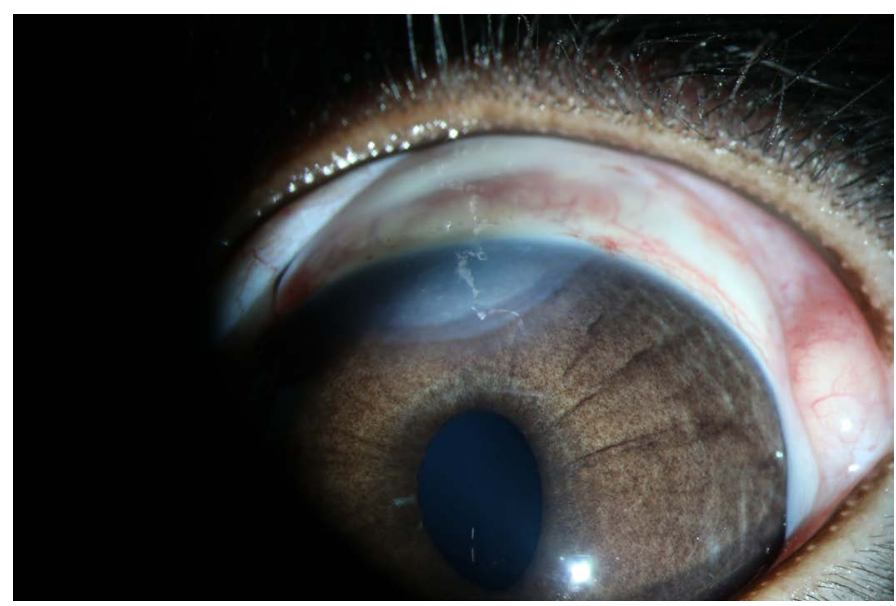

Figure 15. Experimental group: 5 days after thermal burn

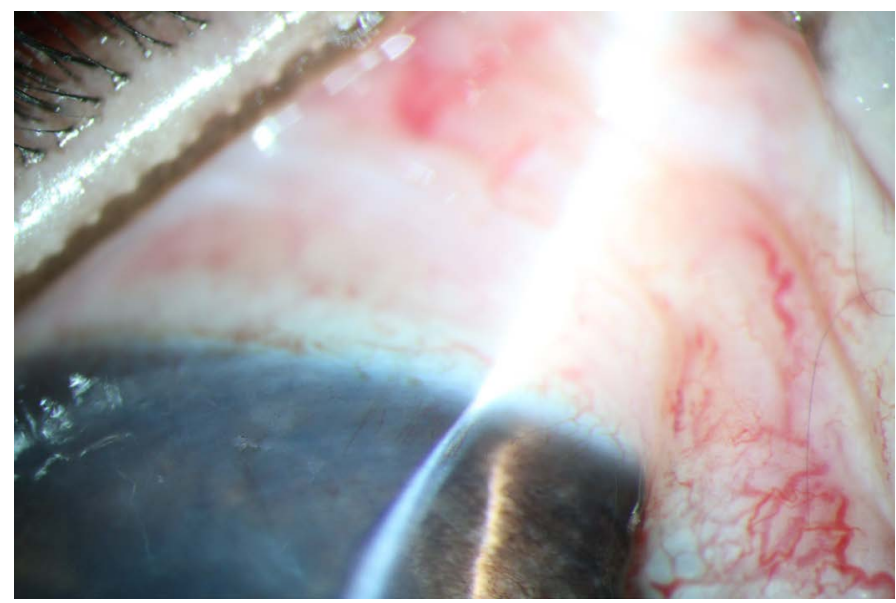

Figure 16. Control group: 5 days after thermal burn 


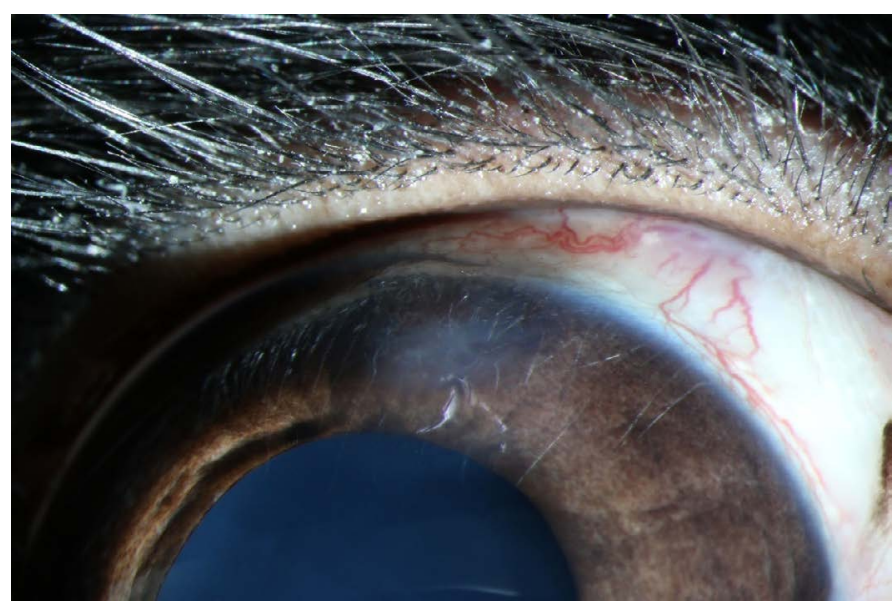

Figure 17. Experimental group: 30 days after thermal burn.

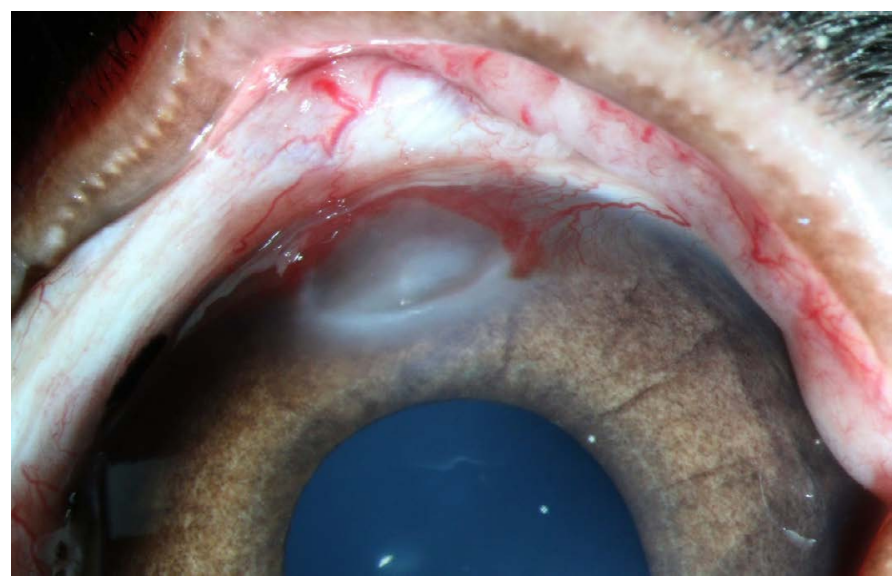

Figure 18. Control group: 30 days after thermal burn

increase in the thickness of the cornea. This minor swelling was absent on the third day.

Within a week the parameters of thickness of the cornea between the control and experimental groups were compared. The cornea did not return to its initial thickness in the zone of thermal injury by the $30^{\text {th }}$ day in the control group. However, the same parameters in the animals belonging to the experimental group exceeded those in the initial stages.

\section{Histological results}

$7^{\text {th }}$ day - experimental group (A): perinuclear swelling in the basal epithelium of the cornea, Bowman's membrane - no visible changes, stroma - no visible changes, Descemet's membrane - no visible changes, loss in the cell density of the corneal endothelium, the presence of precipitates cannot be excluded. Hematoxylin and eosin dye, observed at 100x magnification. (Figure 19)

$7^{\text {th }}$ day - control group (B): The epithelium has been eroded due to necrosis and inflammation, Bowman's membrane differentiated without visible changes, stroma - no visible changes, Descemet's membrane slightly thicker, loss in the cell density of the corneal endothelium, the presence of precipitates cannot be excluded. Hematoxylin and eosin dye, observed at 100x magnification. (Figure 20)

$3^{\text {th }}$ day - experimental group (A): the structural integrity of the cornea remained intact, loss in the cell density of the corneal endothelium. Hematoxylin and eosin dye, observed at 100x magnification. (Figure 21)

$3^{\text {th }}$ day - control group (B): abundant vascular ingrowth in the anterior layers of the cornea, formation of a vascular cataract, persistent swelling of the stroma. Hematoxylin and eosin dye, observed at 100x magnification. (Figure 22)

\section{Histological results of the $3^{\text {rd }}$ stage of the experiment}

Significant changes in both the control and experimental groups were not observed on the first day. Blepharospasms and lacrimation was observed in both groups along with edema and hyperaemia in the conjunctiva of all the eyes. There was an observable decrease in the transparency as a result of a moderate edema in the superficial layers of the stroma.

Experimental group, $\mathbf{1}^{\text {st }}$ day: H\&E staining 200x magnification. Observable disruption in the structure of the epithelia. Loss in the cell density of the stroma due to edema, appearance of blood vessels in the cornea and injury in the endothelium, and detachment of Descemet's membrane. (Figure 23)

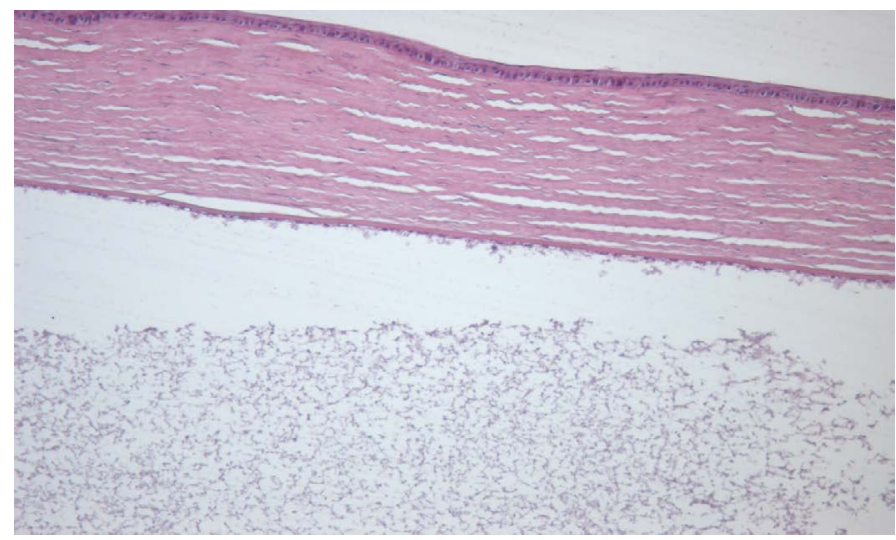

Figure 19. $7^{\text {th }}$ day - experimental group (A)

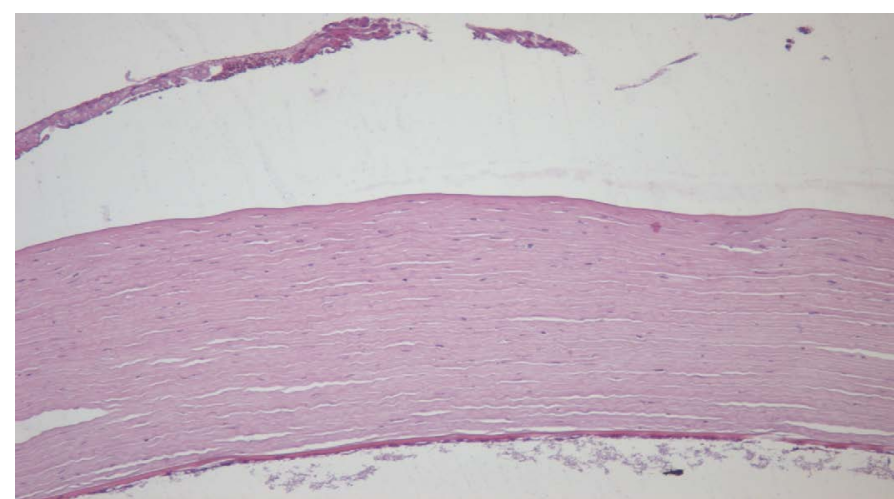

Figure 20. $7^{\text {th }}$ day - control group (B)

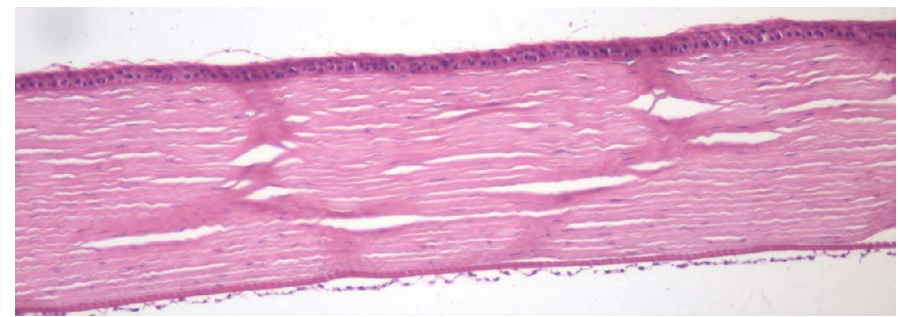

Figure 21. $30^{\text {th }}$ day - experimental group (A) 


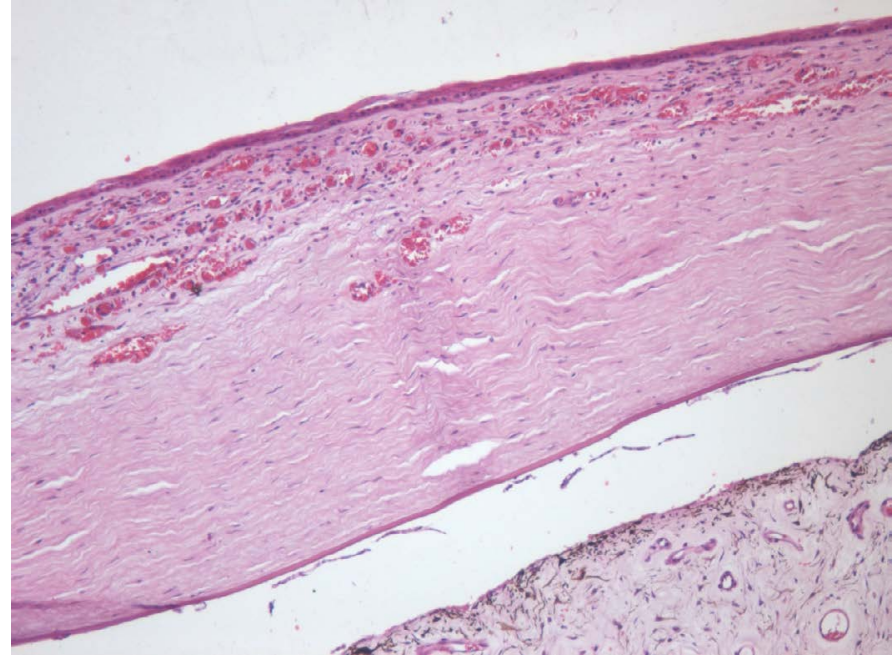

Figure 22. $30^{\text {th }}$ day - control group (B)

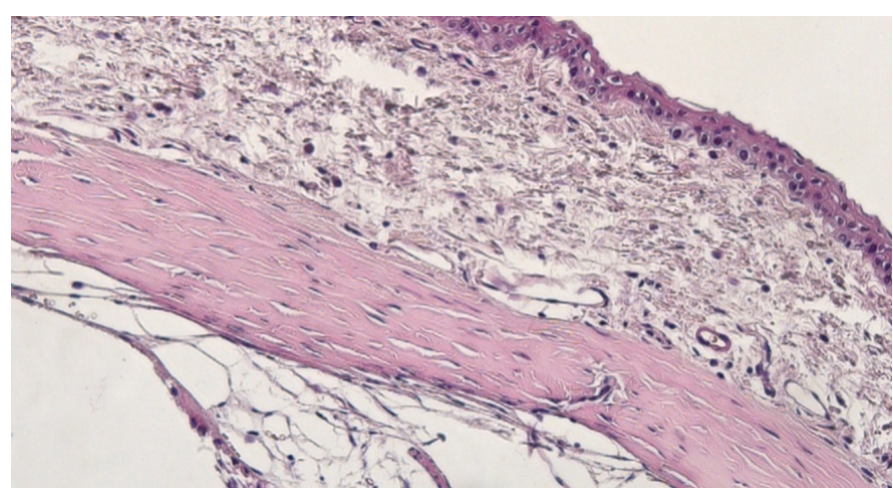

Figure 23. Experimental group, $1^{\text {st }}$ day

Control group, $1^{\text {st }}$ day: H\&E staining 200x magnification. Observable disruption in the structure of the epithelia. Thinning of the stoma was observed in the regions exhibiting deepithelialization. The loss in cell density of the stroma as a result of edema, newly formed blood vessels in the cornea and injury in the endothelium, and detachment of Descemet's membrane. (Figure 24)

On the $5^{\text {th }}$ day visible signs of inflammation were observed in the experimental group. Edema and hyperaemia developed in the conjunctiva of all the eyes belonging to the control group.

Experimental group, $5^{\text {th }}$ day: H\&E staining, 100x maginification. Restoration of the structural integrity of the epithelial and endothelial layers. Decrease in the number of cells in the epithelium. Единичные участки разволокнения стромы. Formation of vessels in the cornea. (Figure 25)

Control group, $\mathbf{5}^{\text {th }}$ day: H\&E staining, 100x magnification. Erosion of the epithelium, injury in the superficial cellular layers. Appearance of vessels in the stroma, loss in cell density due to edema. Precipitation on the endothelium. (Figure 26)

On the $21^{\text {st }}$ day, blood vessels were isolated (largest during the beginning of the experiment) in the cornea of the experimental group. The vasculature in the control group was more extensive.

Experimental group, $2^{\text {st }}$ day: Perilimbal zone. H\&E staining, 100x magnification. Structure of the cornea has been completely restored. A single blood vessel can be observed. No signs of damage can be seen in the endothelium. (Figure 27)

Control group, $21^{\text {st }}$ day: Perilimbal zone. H\&E staining, 100x magnification. Cells of the epithelium showed a tendency towards recovery. Loss of cell density in the cornea as a result of the newly formed blood vessels with a large diameter. The structure of the endothelium had restored. (Figure 28)

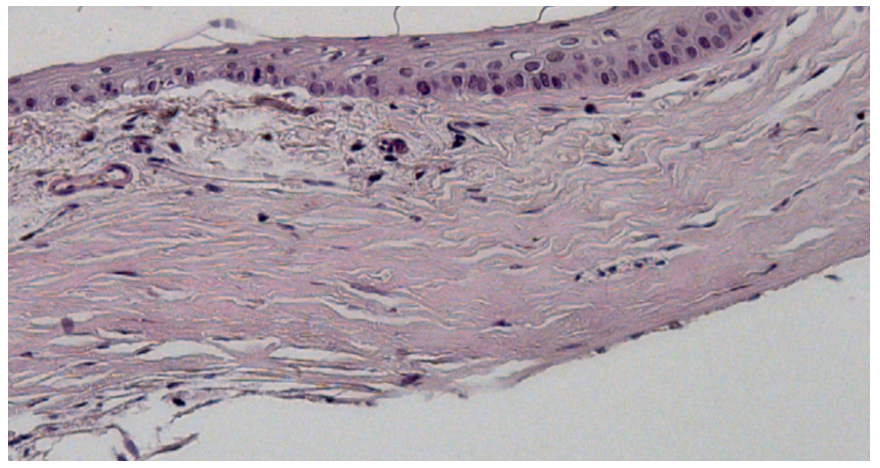

Figure 24. Control group, $1^{\text {st }}$ day

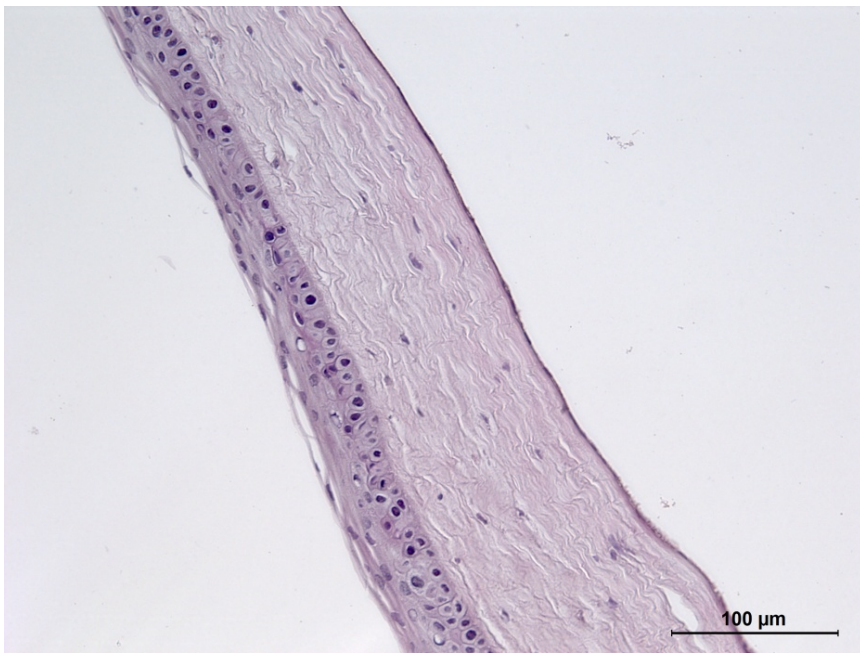

Figure 25. Experimental group, $5^{\text {th }}$ day

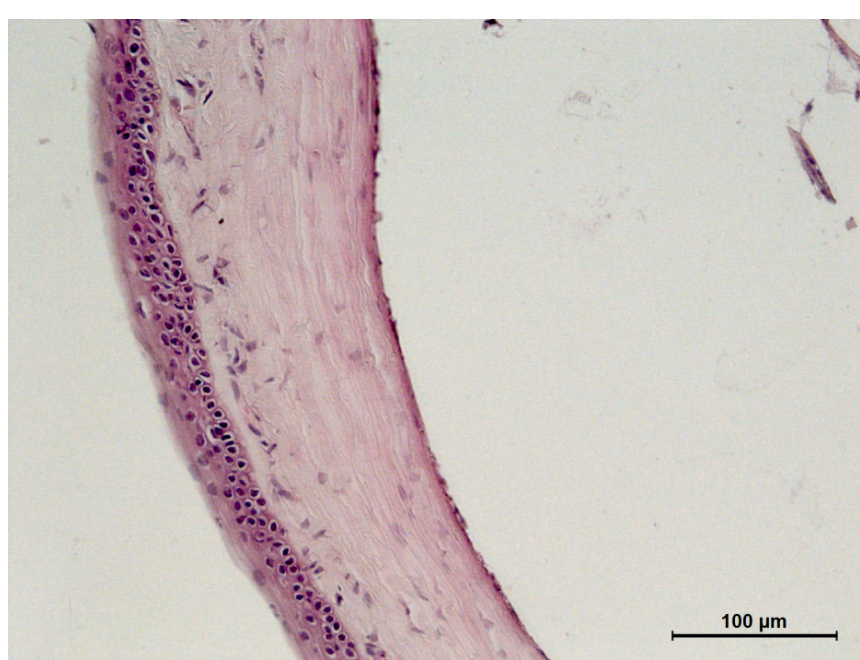

Figure 26. Control group, $5^{\text {th }}$ day 


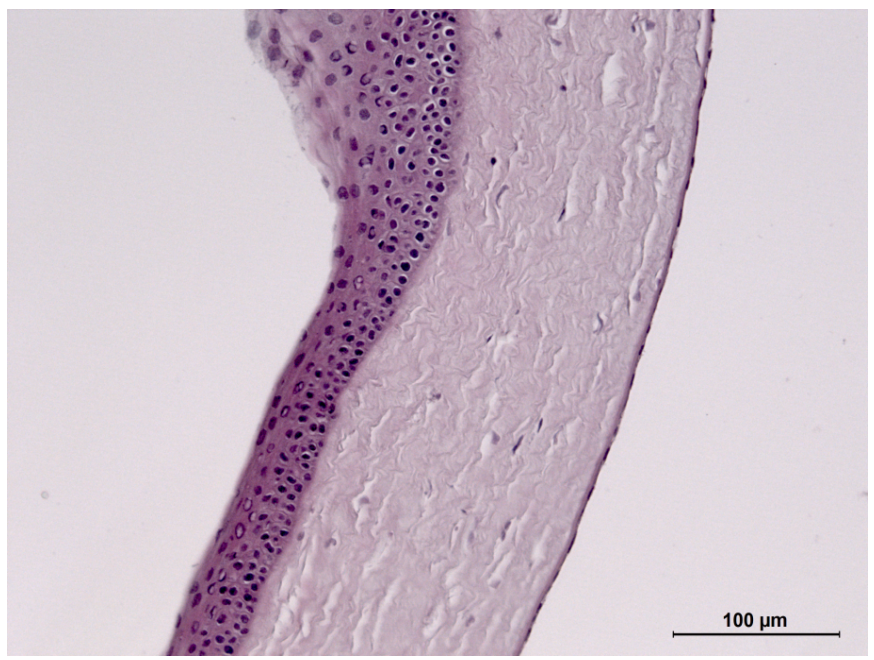

Figure 27. Experimental group, $21^{\text {st }}$ day

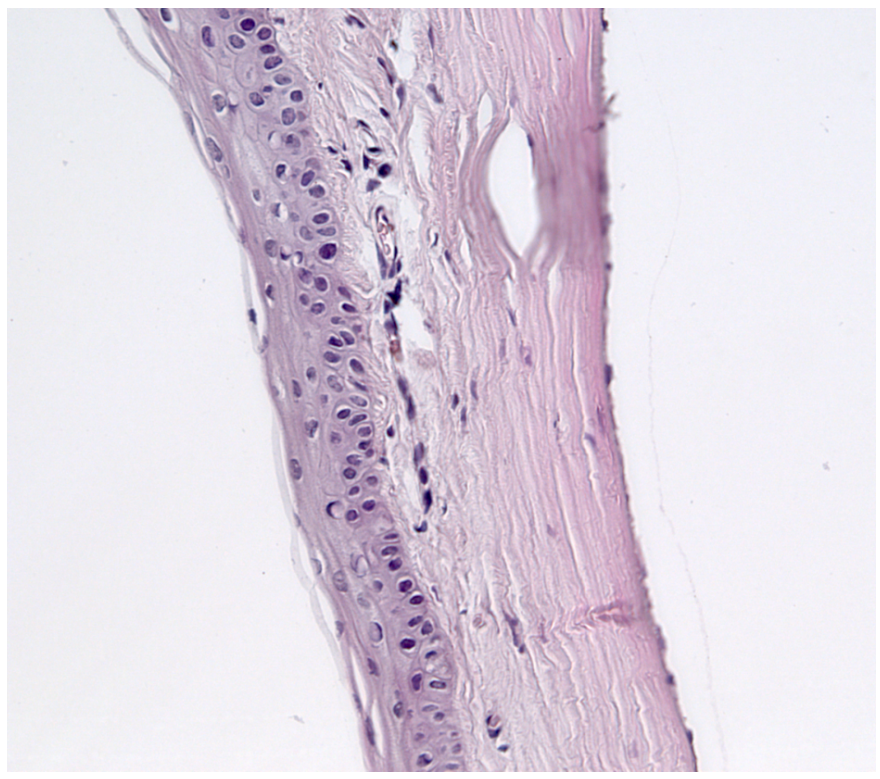

Figure 28. Control group, $21^{\text {st }}$ day

\section{Discussion}

Our research shows that the conditioned medium and lysate of MSC have protective properties in the treatment of thermal injury and keratitis of the cornea and keratitis of the cornea. We observed a decrease in inflammatory response which caused a reduction in swelling, a faster recovery period of the corneal surface, and inhibition of neovascularization. All these factors ultimately led to faster restoration in the optical properties of the eye.

It should be noted that the medium used had a high concentration of VEGF (upto $1200 \mathrm{pg} / \mathrm{mL}$ ) as is shown in our previous work [7]. Despite such high concentrations, we observed suppression of neovascularization in the cornea. This either might be due to the inhibition of VEGF receptors as a result of a high ligand concentration (peptide solution), or because of other factors secreted by stem cells. [8-22]

\section{Conclusions}

After analyzing the statistical data, based on the graphs included, it can be concluded that the use of peptides facilitates tissue repair.
Our results present the prospect of using peptide solutions for the treatment of keratitis and thermal burns of the cornea upto a diameter of $8 \mathrm{~mm}$ with or without damage to the limbal tissue. Further research into the optimization of the current method in treating diseases of the cornea by using different concentrations of the peptide solution and administering them in combination with other drugs.

\section{Funding information}

The research was conducted with the support of Russian Foundation for basic Research (RFBR) grant No. 17-07-00439 «Evaluation of the effectiveness of treatment of acute liver failure by morphometric analysis of hepatocyte damage in the experiment».

\section{References}

1. Kerimov KT, Djafarov AI, Gahramanov FS (2005) Burns of the eyes. Pathogenesis and treatment. Moscow: Publishing House of RAMS.

2. Makarov PV, Gundorova RA, Slepova OS (2000) Complications of severe burn injury of the eye and their surgical prophylaxis / All-Russian Congress of Ophthalmologists. Tez Dokl Moscow. 87.

3. Sharifipour F, Baradaran-Rafii A, Idani E, Zamani M, Jabbarpoor Bonyadi MH (2011) Oxygen therapy for acute ocular chemical or thermal burns: a pilot study. $\mathrm{Am} \mathrm{J}$ Ophthalmol 151: 823-828. [Crossref]

4. Khubutiya MS, Vagabov AV, Temnov AA, Sklifas AN (2014) Paracrine mechanisms of proliferative, anti-apoptotic and anti-inflammatory effects of mesenchymal stromal cells in models of acute organ injury. Cytotherapy. 16: 579-585

5. Tereshenko A.V, Beliy YA, Takhchidi EK, Novikov SV, Maichook NV (2015) Experimental study of the influence of $0.1 \%$ solution of benzalkonium chloride on the cornea in rabbits. Bulletin of the Orenburg State University.

6. Yagi H, Soto-Gutierrez A, Navarro-Alvarez N, Nahmias Y, Goldwasser Y, et al. (2010) Reactive bone marrow stromal cells attenuate systemic inflammation via sTNFR1. Mol Ther 18: 1857-1864. [Crossref]

7. Lee RH, Oh JY, Choi H, Bazhanov N (2011) Therapeutic factors secreted by mesenchymal stromal cells and tissue repair. J Cell Biochem 112: 3073-3078. [Crossref]

8. K.T. Kerimov, A.I. Djafarov, F.S. Gahramanov (2005) Burns of the eyes. Pathogenesis and treatment. Moscow: Publishing House of RAMS.

9. Kashnikov VV (2001) Modern aspects of rehabilitation of victims in emergency situations. Ophthalmic surgery and ter 21: $38-41$

10. Kovanov VV, Sycennikov IA (1978) Collagenoplasty in medicine. C 375

11. Polunin GS (1977) Proteolytic enzymes and their inhibitors in ophthalmology. Bulletin of Ophthalmology. 3: 79-81

12. Mozhaev GA, Zabolotniy VN, Dyakonov VP (1995) Emergency medical aid to victims of accidents and catastrophes. Kiev.

13. Gallyullina R.Sh (1992) Keratoamnioplasty: Dis.Candidate of Medicine.

14. Brikman IV, Ibadova SI, Kotelyansky VE, Fibrokinetin (1986) A new class of medicinal substances in the treatment of epithelial defects of the cornea. Pathophysiology and biochemistry of the eye. 88-92.

15. Babich GA, Zelenskaya MV (1989) The use of soft contact lenses, saturated with drugs, with disease of the organ of vision / Actual questions of contact correction of sight. $45-50$

16. Iakimenko SA, Buznyk OI, Rymgayllo-Jankowska B (2013) Transplantation of the amniotic membrane in the treatment of recurrent ulcers of the cornea, after severe chemical and thermal eye trauma. Eur J Ophthalmol 23: 496-503.

17. Dobrowolski D, Wylegala E, Orzechowska-Wylegala B, Wowra B, WróblewskaCzajka E (2011) Application of autologous cultivated corneal epithelium for corneal limbal stem cell insufficiency--short-term results. Klin Oczna 113: 346-351. [Crossref]

18. Avunduk AM, Tekelioglu Y (2006) Therapeutic use of limbal stem cells. Curr Stem Cell Res Ther 1: 231-238. [Crossref] 
Tereshenko A (2018) Methods of treating keratitis and thermal burns in the cornea at various locations and areas with ligand-based peptides

19. Shimazaki J, Konomi K, Shimmura S, Tsubota K (2006) Ocular surface reconstruction for thermal burns caused by fireworks. Cornea 25: 139-145. [Crossref]

20. Jurowski P, GoĹ> R, Owczarek G, Gralewicz GZ (2005) Corneal endothelial cells' protection against thermal injury: influence of ophthalmic viscoelastic substances in experimental study on rabbits. Eur J Ophthalmol 15: 674-679. [Crossref]
21. Stoiber J, Ruckhofer J, Muss W, Grabner G (2002) [Amniotic membrane transplantation with limbal stem cell transplantation as a combined procedure for corneal surface reconstruction after severe thermal or chemical burns]. Ophthalmologe 99: 839-848. [Crossref]

22. Prockop DJ, Oh JY (2012) Mesenchymal stem/stromal cells (MSCs): role as guardians of inflammation. Mol Ther 20: 14-20. [Crossref]

Copyright: $(\mathbb{C} 2018$ Tereshenko A. This is an open-access article distributed under the terms of the Creative Commons Attribution License, which permits unrestricted use, distribution, and reproduction in any medium, provided the original author and source are credited. 